\title{
Analysis and Applications of Location-Aware Big Complex Network Data
}

\author{
Jianxin Li $\mathbb{D},{ }^{1}$ Ke Deng, ${ }^{2}$ Xin Huang $\mathbb{D},{ }^{3}$ and Jiajie $\mathrm{Xu}^{4}$ \\ ${ }^{1}$ Deakin University, Australia \\ ${ }^{2}$ RMIT University, Australia \\ ${ }^{3}$ The Hong Kong Baptist University, Hong Kong, China \\ ${ }^{4}$ Soochow University, China \\ Correspondence should be addressed to Jianxin Li; jianxin.li@deakin.edu.au
}

Received 10 July 2019; Accepted 10 July 2019; Published 14 July 2019

Copyright (C) 2019 Jianxin Li et al. This is an open access article distributed under the Creative Commons Attribution License, which permits unrestricted use, distribution, and reproduction in any medium, provided the original work is properly cited.

In response to the ever-increasing challenges of locationaware network data like spatiosocial network and traffic network data, the network data processing technology is experiencing revolutionary changes in each stage including data collecting, cleaning, organizing, interpreting, analyzing, utilizing, and visualization. Those changes lead to a globally noticeable development trend of the convergence with big data frameworks, network analytical modeling, link or route prediction, and recommendation systems. This special issue aims at providing a forum to present recent advancements in the convergent research about big complex network data. Challenges include real-time event detection in a city, congestion discovery in a traffic network, location prediction of social users, social users' behavior recognition in physical world, and unified systems of processing multidimensional complex network data. The robust solutions call for highly innovative techniques in the fields including, but not limited to, machine learning, genetic algorithms, chaos, genetic algorithms, cellular automata, neural networks, and evolutionary game theory.

The special issue has attracted high-quality submissions from researchers worldwide in the areas of machine learning, artificial intelligence, data mining, natural language processing, data and web mining, and big data management to utilize their expertise and match up to the challenges of developing more efficient and practical algorithms or models to obtain smart knowledge from daily generated invaluable social network data and traffic network data. The total of submissions is 40. After single-blind peer review by at least two reviewers, 19 papers were finally accepted to be published. The accepted rate is $47.5 \%$. The average number of authors for each accepted paper is 4.2. The affiliated institutes of authors are from China, Australia, France, India, Saudi Arabia, and Canada. These accepted papers can be organized in different groups. The focus of the first group of articles is location-based social network research. The paper titled "Targeted Influential Nodes Selection in Location-aware Social Networks" by S. Yang et al. presented an effective solution to identify the target based influential nodes in location-aware social networks. The paper titled "Discovering Travel Community for POI Recommendation on Location-Based Social Networks" by L. Tang et al. improved the community detection method for high-quality point-of-interest recommendation. The other three papers- “A Joint Deep Recommendation Framework for Location-Based Social Networks" by O. Tal and Y. Liu, "Optimal Proxy Selection for Socioeconomic Status Inference on Twitter" by J. L. Abitbol et al., and "Incremental Bilateral Preference Stable Planning over Event Based Social Networks" by B. Li et al.- invented the new machine learning technologies to explore the useful annotations for users and recommendation. The focus of the second group of articles is traffic based road network research. The papers titled "An Effective Algorithm for Video-Based Parking and Drop Event Detection" by G. Li et al., "Location-Aware Web Service Composition Based on the Mixture Rank of Web Services and Web Service Requests" by J. Lu et al., and "Predicting 
Quality of Service via Leveraging Location Information" by L. Chen et al. provided the effective solution to discover the important location of delivering high-quality service in smart city environment. The other three papers titled "SemanticAware Top-k Multirequest Optimal Route" by S. Wang et al., "A Novel Index Method for K Nearest Object Query over Time-Dependent Road Networks" by Y. Yang et al., and "A Destination Prediction Network Based on Spatiotemporal Data for Bike-Sharing" by J. Jiang et al. proposed efficient algorithms and models to choose the optimal route planning. The focus of the third group of articles is deep learning. The papers titled "Sign Prediction on Unlabeled Social Networks Using Branch and Bound Optimized Transfer Learning" by W. Yuan et al., "Cognitive Driven Multilayer Self-Paced Learning with Misclassified Samples" by Q. Zhu et al., and "A Block Object Detection Method Based on Feature Fusion Networks for Autonomous Vehicles" by Q. Meng et al. investigated the deep learning technologies to optimize the entity recognition in social network data. The other five papers titled "pSPARQL: A Querying Language for Probabilistic RDF Data" by H. Fang, "Edge Computing in an IoT Base Station System: Reprogramming and Real-Time Tasks" by H. Wu et al., "Finding the Shortest Path with Vertex Constraint over Large Graphs" by Y. Yang et al., "Evaluation of Residential Housing Prices on the Internet: Data Pitfalls" by $\mathrm{M}$. Li et al., and "Promoting Geospatial Service from Information to Knowledge with Spatiotemporal Semantics" by J. Geng et al. contributed a diverse range of topics using the semantic information. The solutions proposed in these research works include transfer learning, edge computing, data index structure, self-space learning, video analysis, feature fusion networks, multilayer classification, spatiotemporal pattern recognition, and probabilistic resource description framework data analysis. The effectiveness of the proposed solutions to the targeted problems has been reported based on empirical study and/or analysis.

In summary, the research papers cover a wide range of applications including geospatial services, location-based social network recommendation, social network influential node selection, data pitfall detection for residential house price prediction, socioeconomic status inference, parking detection, object detection for autonomous vehicle, service quality prediction, bike-sharing, label prediction in social networks, $\mathrm{k}$ nearest neighbor query over time-dependent road networks, and IoT base station system optimization.

\section{Conflicts of Interest}

The authors declare that they have no conflicts of interest.

\section{Acknowledgments}

This work has been funded by the Australia Research Council Discovery Program under Grant No. DP160102114.

Jianxin Li

Ke Deng

Xin Huang

Jiajie Xu 


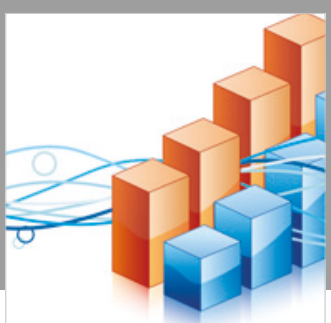

Advances in

Operations Research

\section{-n-m}
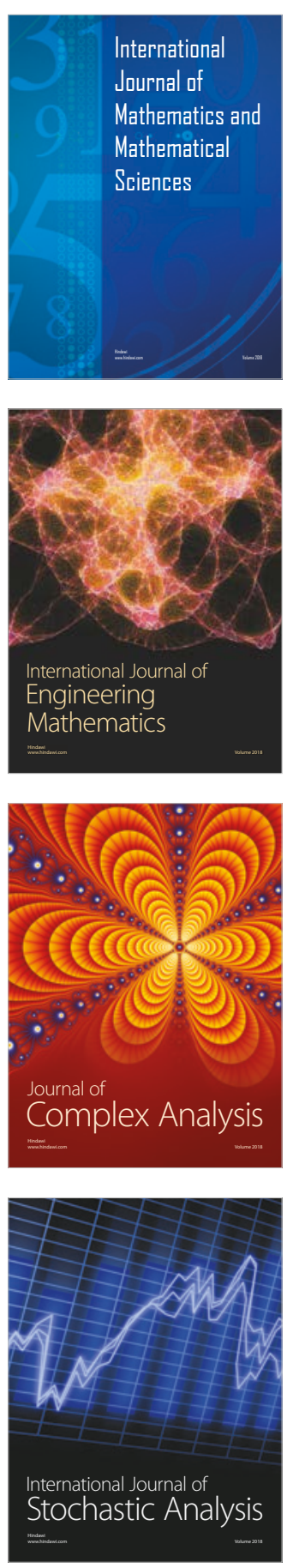
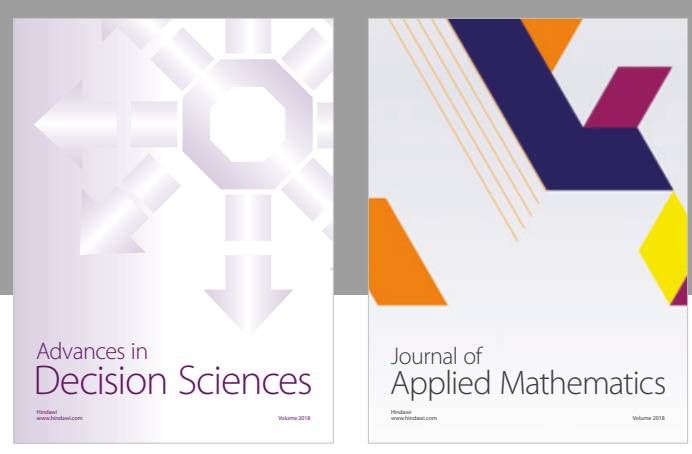

Journal of

Applied Mathematics
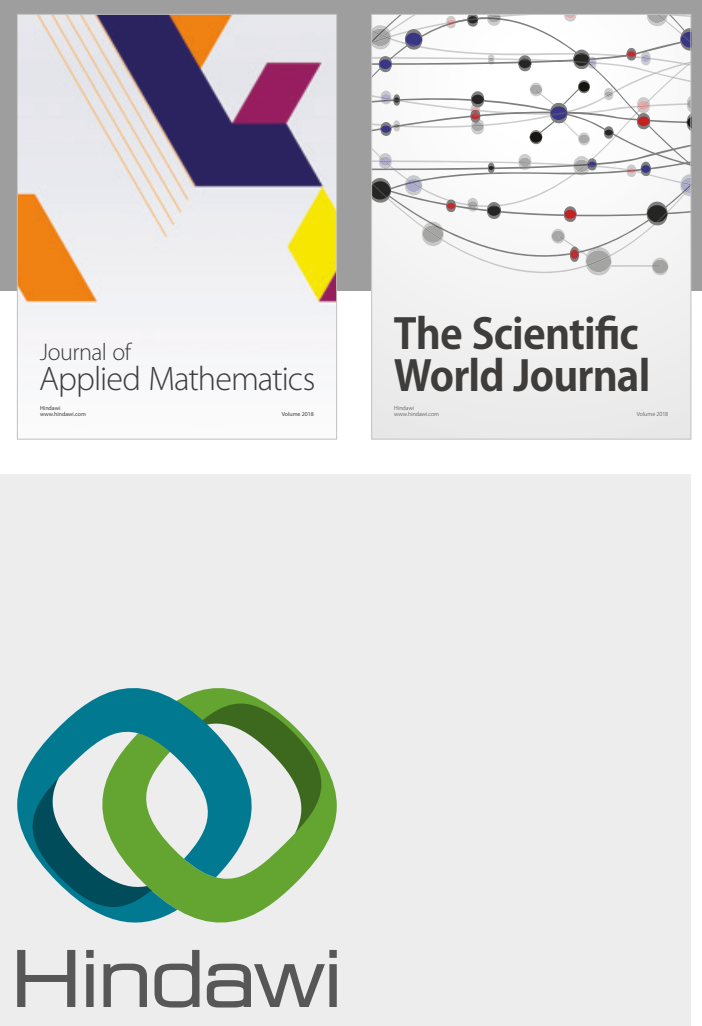

Submit your manuscripts at

www.hindawi.com

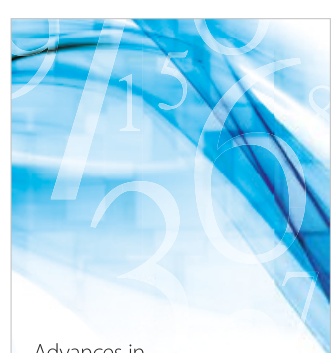

Advances in
Numerical Analysis
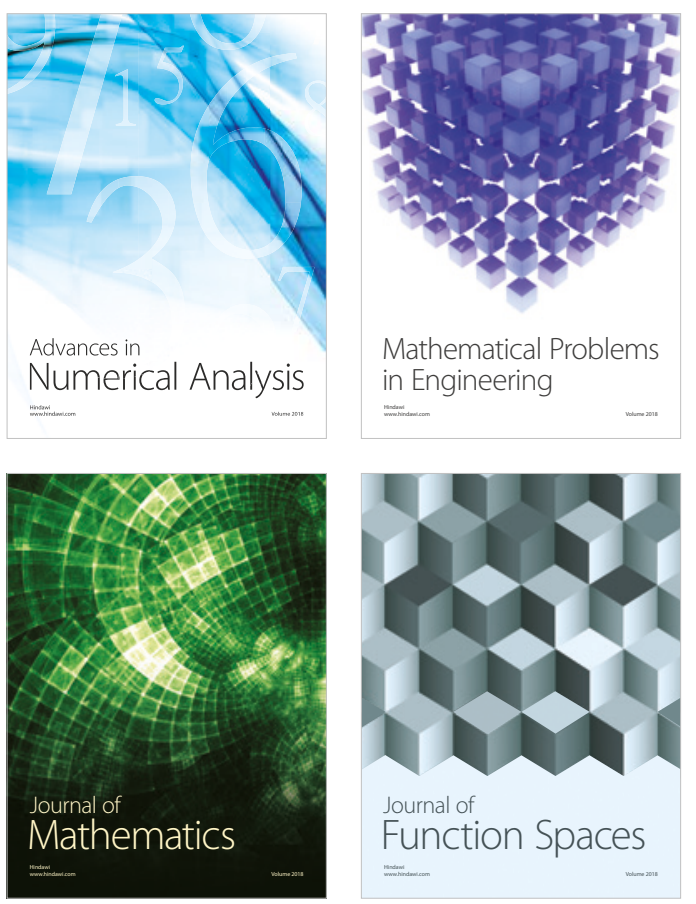

Mathematical Problems in Engineering

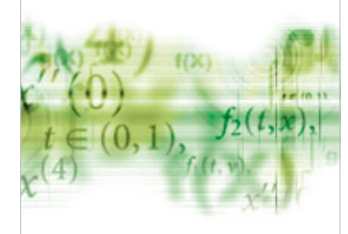

International Journal of

Differential Equations

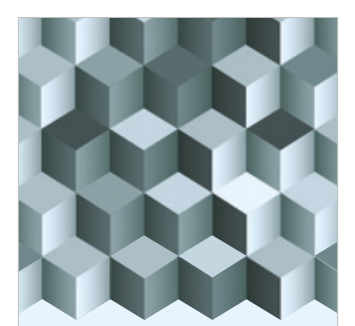

Journal of

Function Spaces
The Scientific

World Journal

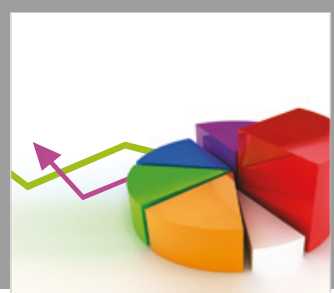

Journal of

Probability and Statistics
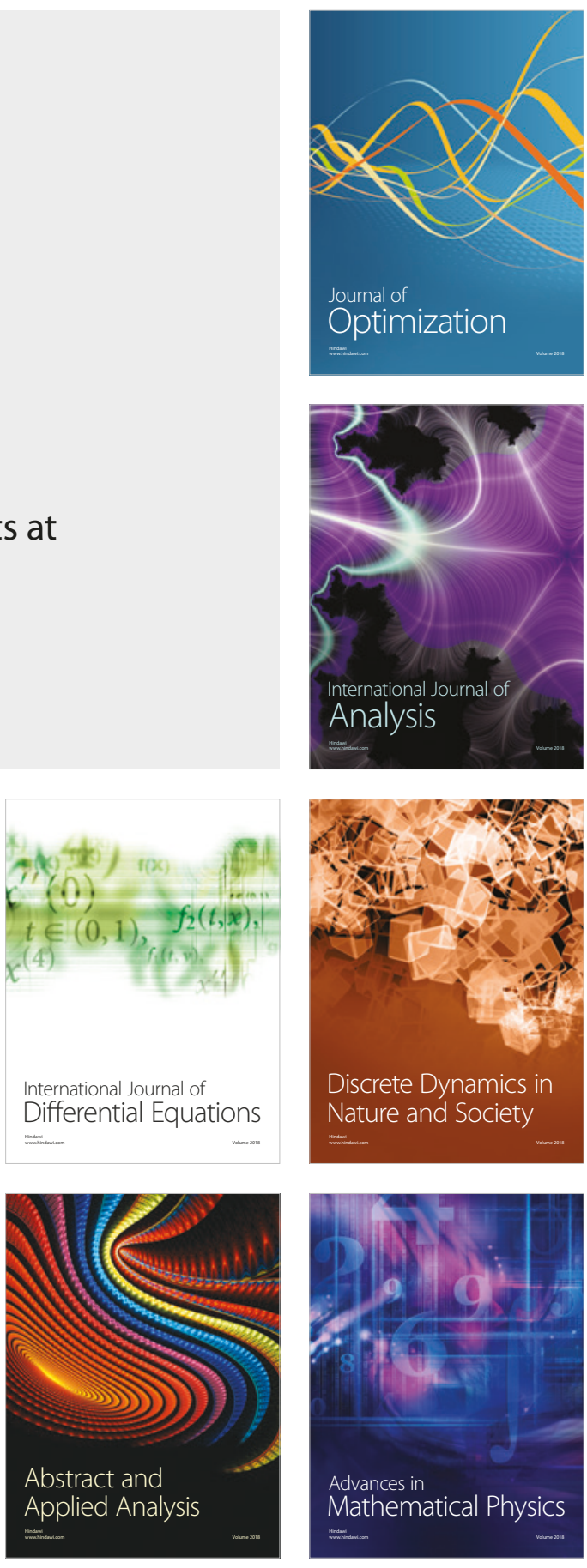